\title{
Atomic-scale Mechanisms of Defect-Induced Retention Failure in Ferroelectric Materials
}

\author{
L. Li $^{1}$, L. Xie ${ }^{1}$, Y. Zhang ${ }^{1}$, J. R. Jokisaari ${ }^{1}$, H.M. Christen ${ }^{2}$, and X. Q. Pan ${ }^{3, *}$ \\ ${ }^{1}$ Department of Materials Science and Engineering, University of Michigan, Ann Arbor, MI \\ 48109, United States \\ ${ }^{2}$ Materials Science and Technology Division, Oak Ridge National Laboratory, Oak Ridge, TN \\ 37831, United States \\ ${ }^{3}$ Department of Chemical Engineering and Materials Science and Department of Physics and \\ Astronomy, University of California - Irvine, Irvine, CA 92697, US
}

The ease of reversibly switching the spontaneous polarization of a ferroelectric with an applied electric field has made this material attractive for application of high-density nonvolatile memories. One of the major challenges impeding this application, however, has been the socalled "retention failure" phenomenon - a self back-switching process of the written polarization that can lead to data loss. An understanding of the atomic-scale mechanism of the retention failure process is thus necessary to engineer reliable ferroelectric devices. Here, using in situ transmission electron microscopy (TEM), we report direct observation of polarization backswitching induced by non-stoichiometric defects that commonly exist in ferroelectrics. Our results of atomic-resolution scanning transmission electron microscopy (STEM) show a novel mechanism of the retention failure process, revealing that the process is induced by the strong atomic interaction between the defects and the surrounding domains.

$\mathrm{BiFeO}_{3}$ (BFO) thin film of $50 \mathrm{~nm}$ in thickness with $8 \mathrm{~nm}$ thick $\mathrm{La}_{0.7} \mathrm{Sr}_{0.3} \mathrm{MnO}_{3}$ (LSMO) bottom electrode was grown on $\mathrm{LaAlO}_{3}$ substrate by pulsed laser deposition. In this film, BFO possesses a tetragonal-like ( $T$-like) structure (Fig. 1a) in most regions, and a large density of nonstoichiometric defects is observed within the $15 \mathrm{~nm}$ thick region above the BFO/LSMO interface. Local switching was performed upon applying a bias between a tungsten surface probe and the LSMO bottom electrode (Fig. 1b). The bias was linearly increased from 0 to $10 \mathrm{~V}$ during $20 \mathrm{~s}$ and then removed. A plot of the measured switched domain area versus time during the switching and back-switching process is shown in Fig. 1c. Corresponding selected TEM images in Fig. 1d show the formation of a large "up" polarized domain in the "down" polarized matrix during the initial 0 to $-10 \mathrm{~V}$ ramp and back-switching to a much smaller stable domain pinned by defects after the removal of the bias. To understand the underlying mechanism of the backswitching process, atomic resolution high-angle annular dark-field (HAADF) STEM was performed to image a polarized BFO region interacting with a defect, as shown in Fig 2a. Atomic displacements of $\mathrm{Fe}$ cations from the center of four $\mathrm{Bi}$ neighbors for $\mathrm{BFO}$, which are proportional to the polarization, [1] are measured and shown in Fig. 2b. Due to a strong atomic interaction between the $\mathrm{BFO}$ and the defect, significantly enhanced out-of-plane polarizations were observed for the first BFO lattice layer that is in direct contact with the defect. Such strong interaction would cause a large downward built-in field pointing to the defect and thus destabilize the "up" polarized written domain and cause retention failure.

In conclusion, using in situ TEM combined with atomic resolution STEM, we have shown a strong interaction between ferroelectric polarizations and the non-stoichiometric defects, which 
can cause a large built-in field, leading to local polarization enhancement and destabilizing written domains. Our results provide new insights into the critical role of defects on domain configurations and dynamics and suggest a new route to design ferroelectric devices through defect engineering.

References:

[1]. Li, L., et al., Nano Letters, 2013. 13(11) 5218-5223.

[2]. The authors gratefully acknowledge the financial support through DOE grant DoE/BES DEFG02-07ER46416.
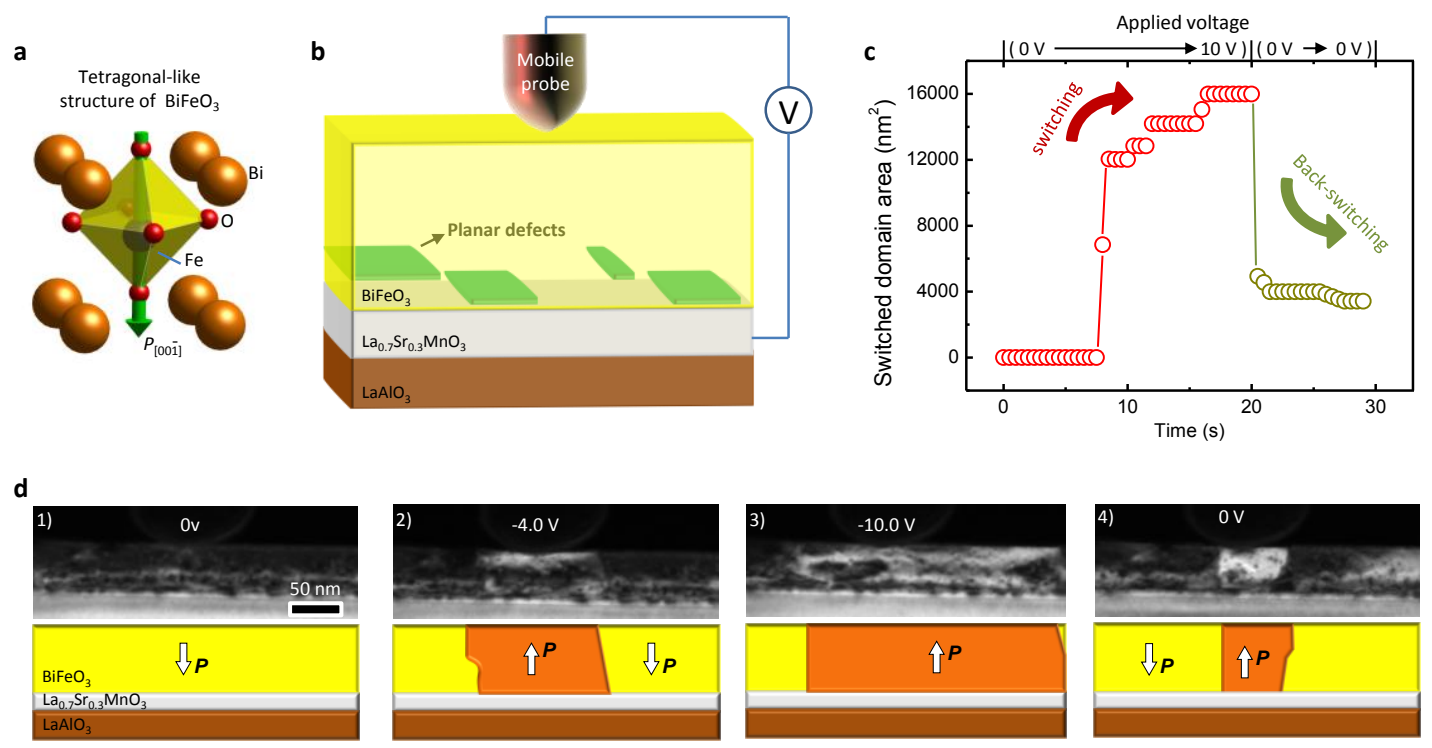

Figure 1. (a) Atomic models of the tetragonal-like structure $\mathrm{BiFeO}_{3}$. (b) Schematic of in situ TEM experimental set-up. (c) Plot of the measured switched domain area versus time extracted from an in situ TEM video. (d) A chronological TEM image series showing the evolution of a written "up" polarized domain in a "down" polarized matrix.
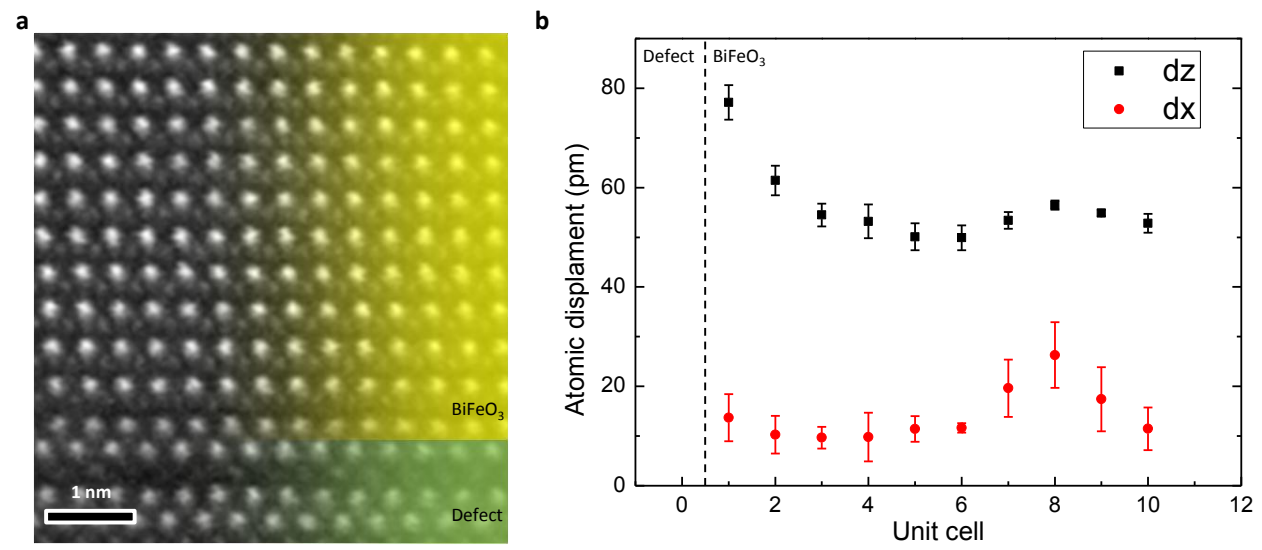

Figure 2. (a) A HADDF STEM image of a polarized BFO region that is in contact with a planar non-stoichiometric defect. (b) Plot of the measured atomic displacements (dz: out-of-plane atomic displacement, dx: in-plane atomic displacement) of Fe cations from the center of four $\mathrm{Bi}$ neighbors for BFO atomic structures shown in (a). 